\title{
THE EFFECT ON FINITE TIME BREAKDOWN DUE TO MODIFIED FOURIER LAWS
}

\author{
BY \\ W. KOSIŃSKI (IPPT, Polish Academy of Sciences) \\ AND \\ K. SAXTON (Loyola University, New Orleans, Louisiana)
}

\begin{abstract}
This paper discusses the finite time blow-up of the amplitude of acceleration waves in the case of heat propagation in one-dimensional rigid and elastic bodies. In both cases dissipation is not strong enough to preserve the smoothness of the solutions whose initial data is far from equilibrium.
\end{abstract}

1. Introduction. Under suitable assumptions on the constitutive relations, the equations of isothermal nonlinear elasticity are of hyperbolic type. This fact can lead to the formation of shocks; that is, the velocity and deformation gradient become discontinuous, and for smooth data the Cauchy problem does not have a global smooth solution (Lax [9]). This strong effect of elastic nonlinearity is due to the fact that there is no dissipation. Many kinds of dissipation, e.g., Dafermos [5], will produce stabilizing phenomena. Such a stabilizing role is played, for example, by heat diffusion. This can be detected by investigating the evolution of the amplitude of acceleration waves along characteristics. Coleman and Gurtin [3, 4] first showed, for inelastic materials with memory, how the amplitude of the waves can approach infinity in finite time.

For a one-dimensional thermoelastic material with heat flux given by Fourier's law, the amplitude of the acceleration waves satisfies a Bernoulli equation, which stays bounded in time only for small initial data (Dafermos [5]). Indeed, Slemrod in [10] proved an existence theorem in thermoelasticity for smooth and small initial data, and Dafermos and Hsiao in [6] showed that, for the large smooth data, solutions of thermoelasticity develop discontinuities in finite time.

In the present paper, the Fourier law modification due to Kosiński [8] has been used and finite speed of heat propagation obtained. The Fourier law for heat flux was modified in a number of papers, for example, by including the time derivative of the heat flux accompanied by a thermal relaxation time (Chester [1]). Another approach is used by Gurtin and Pipkin [7], for rigid conductors, where the constitutive relations are in the form of functionals over the temperature history. An advantage of the 
current approach is in retaining proportionality between heat flux and a form of temperature gradient.

Based on the work by Kosiński [8], we examine the concept of a new temperature scale $\beta$, which is related to the absolute temperature $\vartheta$ by the initial value problem

$$
\begin{gathered}
\beta_{, t}=f(\vartheta, \beta), \\
\beta\left(t_{0}\right)=\beta_{0}, \quad \beta \in(0, \infty) .
\end{gathered}
$$

Instead of the classical proportionality equation for the heat flux $\mathbf{q}=-k \nabla \vartheta$, we assume that

$$
\mathbf{q}=d \nabla \beta,
$$

where $d$, in general, is a function of the deformation $F$ and absolute temperature $\vartheta$.

Constitutive equations for a nonlinear thermoelastic material, with reference mass density $\rho_{0}$, form a set of equations for the following quantities: the Helmholtz free energy $\psi$, specific entropy $\eta$, Piola-Kirchhoff stress tensor $T_{\kappa}$, the heat flux $\mathbf{q}$ in terms of the deformation gradient $F$, the temperature $\vartheta>0$, temperature gradient $\nabla \vartheta$, and the new temperature $\beta$, where

$$
\begin{aligned}
\psi & =\hat{\psi}(F, \vartheta, \nabla \beta), \\
\eta & =\hat{\eta}(F, \vartheta, \nabla \beta)=-\partial_{\vartheta} \hat{\psi}(F, \vartheta, \nabla \beta), \\
T_{\kappa} & =\widehat{T}_{\kappa}(F, \vartheta, \nabla \beta)=\rho_{0} \partial_{F} \hat{\psi}(F, \vartheta, \nabla \beta),
\end{aligned}
$$

and

$$
\mathbf{q}=d \nabla \beta .
$$

Equations (1.3) and (1.1), together, describe the property of the material and state the constitutive relations. Equations (1.3) are compatible with the second law of thermodynamics (there is no dependence of $\nabla \vartheta$ in $(1.3)_{1}-(1.3)_{3}$ ), which is reduced to the following inequality describing the internal dissipation:

$$
-\rho_{0} \partial_{\nabla \beta} \hat{\psi} \cdot \nabla \beta_{, t}-\frac{1}{\vartheta} \mathbf{q} \cdot \nabla \vartheta \geq 0,
$$

where by Eq. $(1.1)_{1}$

$$
\nabla \beta_{, t}=\partial_{\vartheta} f \nabla \vartheta+\partial_{\beta} f \nabla \beta
$$

Using Eqs. (1.4) and (1.2), Kosiński in [8] provides an explicit function $f$ such that the Maxwell-Vernotte-Cattaneo equation holds:

$$
\tau \mathbf{q}_{, t}+\mathbf{q}=-k \nabla \vartheta .
$$

Here $\tau$ is a thermal relaxation time-valued function. We assume (cf. Kosiński [8]) that the free energy function $\hat{\psi}$ is given by

$$
\hat{\psi}(F, \vartheta, \nabla \beta)=\hat{\psi}_{1}(F, \vartheta)+\frac{1}{2} \hat{\varepsilon}_{2}(\nabla \beta)^{2},
$$

where $\hat{\varepsilon}_{2}=k_{0} \tau_{0} /\left(\rho_{0} \vartheta_{0}\right)$ and $\tau_{0}$ and $k_{0}$ are characteristic material constants representing the relaxation time and the thermal conductivity at $\vartheta_{0}$. The additional assumption that $d=$ const $=k_{0}$ is made. 
On using Eqs. (1.1), (1.2), and (1.5), the following results hold (Kosiński [8]):

$$
\begin{gathered}
k=-\tau k_{0} \partial_{\vartheta} f, \quad 1=-\tau \partial_{\beta} f, \\
\tau_{0} f(\vartheta, \beta)=\vartheta_{0} \log \left(\vartheta_{0} / \vartheta\right)+f_{0}(\beta),
\end{gathered}
$$

where

$$
f_{0}(\beta)=\tau_{0} f\left(\vartheta_{0}, \beta\right) .
$$

Motivated by relations (1.7) and (1.8), we will investigate three types of function $f_{0}(\beta)$ proposed in [8]:

$$
\begin{array}{r}
\mathscr{A}: \tau(\beta)=\tau_{0}, \text { then } k(\vartheta, \beta)=k_{0} \vartheta_{0} \vartheta^{-1}, \text { and } \\
f_{0}(\beta)=-\left(\beta-\beta_{0}\right) ;
\end{array}
$$

$\mathscr{B}: \tau(\beta)=\tau_{0} \beta \vartheta_{0}^{-1}$, then $k(\vartheta, \beta)=k_{0} \beta \vartheta^{-1}$, and

$$
f_{0}(\beta)=\vartheta_{0} \log \left(\beta_{0} \beta^{-1}\right) \text {; }
$$

$\mathscr{C}: \tau(\beta)=\tau_{0} \vartheta_{0} \beta^{-1}$, then $k(\vartheta, \beta)=k_{0} \vartheta_{0}^{2}(\beta \vartheta)^{-1}$, and

$$
f_{0}(\beta)=-\frac{1}{2} \vartheta_{0}^{-1}\left(\beta^{2}-\beta_{0}^{2}\right) \text {. }
$$

2. Derivation and hyperbolicity of the heat equation. If we neglect mechanical coupling (i.e., assuming the material is rigid) energy balance takes the form (Kosinski [8])

$$
\rho_{0} c_{V} \vartheta_{, t}+\frac{k_{0} \tau_{0}}{\vartheta_{0}} \nabla \beta \cdot \nabla \beta_{, t}+k_{0} \Delta \beta=\rho_{0} r .
$$

Here $c_{V}=\vartheta \partial_{\vartheta} \eta>0$ is the specific heat at constant volume and $c_{V}$ is a function of $\vartheta$ only, as a result of assumption (1.6) concerning the energy function, and $r$ is the body heat supply.

The temperature $\vartheta$ can be expressed as a function of $\beta_{, t}$ and $\beta$ using Eqs. (1.1) and (1.8), particularly,

$$
\vartheta=\vartheta_{0} \exp \left\{\frac{1}{\vartheta_{0}}\left(f_{0}(\beta)-\tau_{0} \beta_{, t}\right)\right\}
$$

thus,

$$
\vartheta_{, t}=\exp \left\{\frac{1}{\vartheta_{0}}\left(f_{0}(\beta)-\tau_{0} \beta_{, t}\right)\right\}\left(f_{0, \beta} \beta_{, t}-\tau_{0} \beta_{, t}\right) .
$$

Therefore from Eq. (2.1) we obtain a second-order partial differential equation for $\beta$ of the form

$$
-C\left(\beta, \beta_{, t}\right) \beta_{, t t}+b \nabla \beta \cdot \nabla \beta_{, t}+a \Delta \beta+H\left(\beta, \beta_{, t}\right)=\rho_{0} \vartheta_{0} r .
$$

Equation (2.4) is a second-order hyperbolic equation and locally has smooth solutions (Cimmelli and Kosiński [2]).

Before we explain the coefficients in this equation, let us introduce new independent variables

$$
\begin{aligned}
& \beta_{, t}=w, \\
& \mathbf{p}=\nabla \beta,
\end{aligned}
$$


which lead us to a quasilinear system replacing Eq. (2.4)

$$
\begin{gathered}
-C(\beta, w) w_{, t}+b \mathbf{p} \cdot \nabla w+a \nabla \cdot \mathbf{p}+H(\beta, w)=\rho_{0} \vartheta_{0} r, \\
\beta_{, t}=w, \\
\mathbf{p}_{, t}-\nabla w=0 .
\end{gathered}
$$

The coefficients in system (2.6) are

$$
\begin{aligned}
C(\beta, w) & =\rho_{0} \tau_{0} \vartheta c_{V}(\vartheta), \\
H(\beta, w) & =\rho_{0} \vartheta c_{V}(\vartheta) f_{0}(\beta) w, \\
a & =k_{0} \vartheta \vartheta_{0}, \\
b & =k_{0} \tau_{0},
\end{aligned}
$$

and, by Eq. (2.2),

$$
\vartheta=\vartheta(\beta, w)=\vartheta_{0} \exp \left\{\frac{1}{\vartheta_{0}}\left(f_{0}(\beta)-\tau_{0} w\right)\right\} .
$$

Note that by Eqs. $(2.7)_{1}$ and $(2.7)_{2}$

$$
\tau_{0} H(\beta, w)=C(\beta, w) f_{0, \beta}(\beta) w .
$$

This system is hyperbolic, i.e., all eigenvalues are real, and the corresponding set of five eigenvectors are linearly independent for all orientations of the wave normal $\mathbf{n}$.

Routine calculations lead to the following equation for the eigenvalues $\lambda$ :

$$
\lambda^{3}\left(C \lambda^{2}+\lambda b \mathbf{p} \cdot \mathbf{n}-a\right)=0 .
$$

These eigenvalues are real if $a / c \geq 0$ (i.e., $k_{0} \vartheta_{0} /\left(\rho_{0} \tau_{0} \vartheta_{0} c_{V}\right) \geq 0$ ), and

$$
\begin{aligned}
& \lambda_{1}=-\frac{b}{2 C} \mathbf{p} \cdot \mathbf{n}-\left(\frac{b^{2}}{4 C^{2}}(\mathbf{p} \cdot \mathbf{n})^{2}+\frac{a}{C}\right)^{1 / 2}, \\
& \lambda_{2}=-\frac{b}{2 C} \mathbf{p} \cdot \mathbf{n}+\left(\frac{b^{2}}{4 C^{2}}(\mathbf{p} \cdot \mathbf{n})^{2}+\frac{a}{C}\right)^{1 / 2}, \\
& \lambda_{3}=\lambda_{4}=\lambda_{5}=0 .
\end{aligned}
$$

Note that in the limit case, when $\tau_{0} \rightarrow 0$ and $\left(\vartheta c_{V}\right)$ is finite, $\lambda_{1} \rightarrow-\infty$ and $\lambda_{2} \rightarrow \infty$. The corresponding five eigenvectors $\gamma_{k}$ are

$$
\begin{aligned}
& \lambda_{1} \smile \gamma_{1}=\left(0,-\lambda_{1}, \mathbf{n}\right), \\
& \lambda_{2} \smile \gamma_{2}=\left(0,-\lambda_{2}, \mathbf{n}\right), \\
& \lambda_{3} \smile \gamma_{3}=\left(1,0, \mathbf{a}_{3}\right), \\
& \lambda_{4} \smile \gamma_{4}=\left(1,0, \mathbf{a}_{4}\right), \\
& \lambda_{5} \smile \gamma_{5}=\left(1,0, \mathbf{a}_{5}\right),
\end{aligned}
$$

where

$$
\mathbf{a}_{i} \cdot \mathbf{n}=0, \quad i=3,4,5 .
$$


If $\tau_{0}=0$ and $\left(\vartheta c_{V}\right)$ is finite, then for all cases $\mathscr{A}, \mathscr{B}$, and $\mathscr{C}$ (see Eqs. (1.9), (1.10), and (1.11)), $\tau(\beta)=0$, and also the constant in Eq. (1.6), $\hat{\varepsilon}=0$. As a consequence, Eq. (2.1) reduces to

$$
\rho_{0} c_{V} \vartheta_{, t}+k_{0} \Delta \beta=\rho_{0} r .
$$

Comparing formula (1.5) where $\tau=0$ with Eq. $(1.3)_{4}$, we find

$$
\nabla \beta=-\frac{k}{k_{0}} \nabla \vartheta
$$

From the evolution Eq. (1.1) and Eq. (1.8) for $\tau=0$, the following relation holds:

$$
\vartheta_{0} \log \frac{\vartheta}{\vartheta_{0}}=f_{0}(\beta) \text {. }
$$

By Eq. (2.15) we can express $\beta$ as a function of $\vartheta$, and using Eq. (2.14), deduce that

$$
\Delta \beta=-\frac{1}{k_{0}} \nabla \cdot(k \nabla \vartheta)=-\frac{1}{k_{0}}(\nabla k \cdot \nabla \vartheta+k \Delta \vartheta),
$$

where $k=k(\vartheta, \beta(\vartheta))$.

We can now replace Eq. (2.13) by the following equation in terms of $\vartheta$ alone:

$$
\rho_{0} c_{V} \vartheta_{, t}-\frac{d k}{d \vartheta}(\nabla \vartheta)^{2}-k \Delta \vartheta=\rho_{0} r
$$

which is a nonlinear parabolic equation. Thus the case $\tau=0$ reduces to a regular Fourier law.

3. Amplitude of the acceleration wave (rigid material). In this section we will consider the propagation of the heat waves in a one-dimensional, homogeneous rigid body. Then system (2.6) takes the form (for $r=0$ )

$$
\begin{gathered}
-C(\beta, w) w_{, t}+b p w_{, x}+a p_{, x}+H(\beta, w)=0, \\
\beta_{, t}-w=0, \\
p_{, t}-w_{, x}=0,
\end{gathered}
$$

where the constants $b$ and $a$ and the functions $C(\beta, w)$ and $H(\beta, w)$ are given by Eqs. (2.7).

Our aim is to show that as the amplitude of the acceleration waves evolves, it blows up in finite time. This contrasts the classical assumption that involves Fourier's law, when the heat equation is of parabolic type, yielding smooth solutions in rigid conductors.

The one-dimensional acceleration wave is a smooth curve $x=\varphi(t)$, with speed of propagation

$$
s(t)=\dot{\varphi}(t),
$$

across which $\beta, w$, and $p$ are continuous functions, but their derivatives are not, i.e., the jumps of the following derivatives are not zero:

$$
\left[w_{, t}\right] \neq 0, \quad\left[p_{, t}\right] \neq 0
$$


but since $\beta_{, t}=w$,

$$
\left[\beta_{, t}\right]=0
$$

and

$$
[w]=[\beta]=[p]=0 .
$$

Calculating directional derivatives along the wave, defined by

$$
\frac{d}{d t}=\frac{\partial}{\partial t}+s \frac{\partial}{\partial x},
$$

for $[\beta],[w]$, and $[p]$, the following relations hold:

$$
\left[\beta_{, x}\right]=0, \quad\left[w_{, x}\right] \neq 0, \quad\left[p,{ }_{x}\right] \neq 0 .
$$

If we use Eqs. (3.3), (3.4), and (3.5) with Eq. (2.3) we can observe that $\vartheta_{, t}$ and $\vartheta_{, x}$ are discontinuous across the wave, particularly,

$$
\begin{aligned}
& {\left[\vartheta_{, t}\right]=-\tau_{0} \exp \left\{\frac{1}{\vartheta_{0}}\left(f_{0}(\beta)-\tau_{0} w\right)\right\}\left[w_{, t}\right],} \\
& {\left[\vartheta_{, x}\right]=-\frac{1}{S}\left[\vartheta_{, t}\right] .}
\end{aligned}
$$

Evaluating system (3.1) across the wave, remembering Eqs. (3.3), (3.4), and (3.5), we find

$$
\begin{aligned}
-C^{+}\left[w_{, t}\right]+b p^{+}\left[w_{, x}\right]+a\left[p_{, x}\right] & =0, \\
{\left[p_{, t}\right]-\left[w_{, x}\right] } & =0,
\end{aligned}
$$

where $C^{+}=C\left(\beta^{+}, w^{+}\right)$and $w^{+}, \beta^{+}, p^{+}$are the values of $w, \beta, p$ for $x=\varphi(t)$. (Note that $w, \beta$, and $p$ are continuous, i.e., $w^{+}=w^{-}, p^{+}=p^{-}, \beta^{+}=\beta^{-}$.) According to Eq. (3.6)

$$
\left[p_{, x}\right]=-\frac{1}{s}\left[p_{, t}\right]=-\frac{1}{s}\left[w_{. x}\right]=\frac{1}{s^{2}}\left[w_{, t}\right]
$$

and the following equation for nonzero $s$ results:

$$
C^{+} s^{2}+b p^{+} s-a=0 .
$$

Next we assume that the wave is propagating into a material that is in a state of rest, that is,

$$
\begin{aligned}
& \beta(x, t)=\beta^{+}=\mathrm{const}>0, \\
& p(x, t)=p^{+}=0, \\
& w(x, t)=w^{+}=0
\end{aligned}
$$

for $\varphi(t) \leq x$. In this case $C^{+}=C\left(\beta^{+}, 0\right)$ and

$$
s^{2}=\frac{a}{C^{+}}=\text {const }=\frac{k_{0} \vartheta_{0}}{\rho_{0} \tau_{0} \vartheta^{+} c_{l}^{+}} .
$$

Let

$$
\alpha(t)=\left[w_{, t}\right](t)
$$

be the amplitude of the acceleration wave. The amplitude evolves in time along the acceleration wave $x=\varphi(t)$ according to a first-order differential equation. In 
order to derive this equation, we differentiate the system (3.1) with respect to $t$ and evaluate it at the wave

$$
-C^{+}\left[w_{, t t}\right]-C_{, w}^{+}\left[w_{, t}^{2}\right]+b\left[p_{, t} w_{, x}\right]+a\left[p_{, x t}\right]+H_{, w}^{+}\left[w_{, t}\right]=0 .
$$

The symbol " + " means that the functions $C$ and $H$ and their derivatives $C_{, w}$ and $H_{, w}$ are evaluated at $w^{+}=0, \beta^{+}=$const .

Using the relation

$$
2 \frac{d}{d t}\left[w_{, t}\right]=\left[w_{, t t}\right]-\dot{s}\left[w_{, x}\right]-s^{2}\left[w_{, x x}\right]
$$

and the constant speed of propagation (3.13), together with Eq. (3.10), we can rewrite Eq. (3.15) first as

$$
-C^{+}\left[w_{, t t}\right]-C_{, w}^{+}\left[w_{, t}\right]^{2}+b\left[p_{, t}\right]\left[w_{, x}\right]+a\left[p_{, x t}\right]+H_{, w}^{+}\left[w_{, t}\right]=0
$$

and then as the following differential equation for $\alpha$ :

$$
\frac{d}{d t} \alpha=\frac{1}{2 C^{+}}\left\{\frac{b}{s^{2}}-C_{, w}^{+}\right\} \alpha^{2}+\frac{H_{, w}^{+}}{2 C^{+}} \alpha .
$$

Equation (3.18) can be simplified into a form suitable for discussion. To do so we recall formula $(3.13)$ for the speed $s$, notation $(2.7)$, relations $(1.7)_{2},(1.8),(2.8)$, and (2.9) and then calculate that

$$
\begin{aligned}
\tau_{0} H_{, w}^{+} & =C^{+} f_{0, \beta}^{+}=-\frac{\tau_{0} C^{+}}{\tau^{+}}, \\
C_{, w}^{+} & =C_{, \vartheta}^{+} \vartheta_{, w}^{+}=-\frac{\tau_{0}}{\vartheta_{0}} C_{, \vartheta}^{+} \vartheta^{+},
\end{aligned}
$$

where by Eq. (2.8)

$$
\vartheta^{+}=\vartheta_{0} \exp \left\{\frac{1}{\vartheta_{0}} f_{0}(\beta)\right\}, \quad \tau^{+}=\tau\left(\beta^{+}\right),
$$

and

$$
\vartheta_{, w}=-\frac{\tau_{0}}{\vartheta_{0}} \vartheta
$$

Combining Eqs. (3.19) and (3.20) with Eq. (3.18) gives

$$
\frac{d}{d t} \alpha-n \tau_{0} \alpha^{2}+\frac{1}{m \tau_{0}} \alpha=0
$$

Here $n$ and $m$ do not depend on $\tau_{0}$ and

$$
n=\frac{1}{\vartheta_{0}}\left(\frac{c_{V, \vartheta}^{+} \vartheta^{+}}{2 c_{V}^{+}}+1\right) .
$$

Also (see Eqs. (1.9), (1.10), and (1.11))

$$
\frac{m}{2}= \begin{cases}1 & \text { in case } \mathscr{A} \\ \beta^{+} / \vartheta_{0} & \text { in case } \mathscr{B}, \\ \vartheta_{0} / \beta^{+} & \text {in case } \mathscr{C} .\end{cases}
$$


Equation (3.21) is of Bernoulli type, which, for $m>0$, has the property that the solution $\alpha(t)$ blows up to $+\infty$ or $-\infty$ in finite time $t_{1}$, if the initial condition $\alpha_{0}=\alpha(0)$ satisfies the inequality

$$
-n \tau_{0} \alpha_{0}+\frac{1}{m \tau_{0}}<0
$$

and

$$
t_{1}=m \tau_{0} \log \frac{m n \alpha_{0} \tau_{0}^{2}}{m n \alpha_{0} \tau_{0}^{2}-1} .
$$

It is easy to verify that $t_{1}$ is a decreasing function of $\tau_{0}$ for fixed $\alpha_{0}$. The magnitude of the relaxing time $\tau_{0}$ in Eq. (3.21) governs which effects dominate in the behaviour of $\alpha(t)$. Small $\tau_{0}$ plays a stabilizing role and prevents $\alpha(t)$ from breaking up, while large $\tau_{0}$ asserts that the time $t_{1}$ is short.

Let us note two limit cases: first as $\tau_{0} \rightarrow \infty$, and the second as $\tau_{0} \rightarrow 0$. If $\tau_{0} \rightarrow \infty$, then $s^{2} \rightarrow 0$ and $t_{1} \rightarrow 0$, and then disturbances do not propagate; $\alpha(t)$ blows up instantly. If $\tau_{0} \rightarrow 0$, then $s^{2} \rightarrow \infty, t_{1} \rightarrow \infty$, and $\alpha(t) \rightarrow 0$ as $t \rightarrow \infty$, which is the case corresponding to the Fourier law.

From Eq. (3.24) it follows, in the case $\mathscr{A}$, for example, that

$$
n \tau_{0} \alpha_{0}>\frac{2}{\tau_{0}} .
$$

The relaxation time is of order $10^{-10}$ for most materials; hence, the last inequality will be satisfied for $\alpha_{0}$ being of order $10^{20}$, which means that in most situations of physical interest blow-up will not occur. A similar observation is true for the cases $\mathscr{B}$ and $\mathscr{C}$.

4. Thermoelastic material. In this section we will consider a one-dimensional thermoelastic material whose mechanical and thermal properties are described by the constitutive equations (1.3) with $d=k_{0}$ and Eqs. (1.1) together with Eqs. (1.7)(1.11). If $v$ and $\dot{v}$ (or $v{ }_{, t}$ ) denote the particle velocity and the acceleration, then with vanishing body force and heat supply, the balance laws for linear momentum and energy reduce to

$$
\rho v_{, t}-T_{\kappa_{,}}=0
$$

and

$$
\rho_{0}\left(\varepsilon+\frac{1}{2} v^{2}\right)_{, t}-\left(v T_{\kappa}-q\right)_{, x}=0 .
$$

Here $\varepsilon$ is the interval energy and $\varepsilon=\psi+\eta \vartheta$. The compatibility condition is

$$
F_{, t}-v_{, x}=0 \text {, }
$$

the constitutive equation for $q$ (cf. Eq. (1.2)) is

$$
q=k_{0} \beta_{, x},
$$

and the evolution equation for $\beta$ is (1.1), with $f(\vartheta, \beta)$ given by (1.8). We can now write the basic system of equations for the one-dimensional thermoelastic body in 
the form

$$
\begin{gathered}
v_{, t}-T_{F}(F, \beta, w) F_{, x}+B(F, \beta, w) w_{, x}-G(F, \beta, w, p)=0, \\
F_{, t}-v_{, x}=0, \\
\beta_{, t}-w=0, \\
-C(F, \beta, w) w_{, t}+D(F, \beta, w) v_{, x}+b p w_{, x}+a p_{, x}+H(F, \beta, w)=0, \\
p_{, t}-w_{, x}=0 .
\end{gathered}
$$

The unknown functions are $(v, F, \beta, w, p)$, where (cf. Eq. (2.5)) $w=\beta_{, t}$, $p=\beta_{, x}$, and

$$
\begin{aligned}
T_{F}(F, \beta, w) & =T_{F}(F, \vartheta)=\partial_{F F} \hat{\psi}_{1}(F, \vartheta) \\
T_{\vartheta}(F, \beta, w) & =T_{\vartheta}(F, \vartheta)=\partial_{F \vartheta} \hat{\psi}_{1}(F, \vartheta) \\
B(F, \beta, w) & =\frac{\tau_{0}}{\vartheta_{0}} \vartheta T_{\vartheta}(F, \vartheta) \\
C(F, \beta, w) & =\rho_{0} \tau_{0} \vartheta c_{V}(F, \vartheta) \\
c_{V}(F, \vartheta) & =\vartheta \partial_{\vartheta} \hat{\eta}=-\vartheta \partial_{\vartheta \vartheta} \hat{\psi}_{1}(F, \vartheta), \\
D(F, \beta, w) & =-\rho_{0} \vartheta \vartheta T_{\vartheta}(F, \vartheta) \\
G(F, \beta, w, p) & =\frac{\vartheta}{\vartheta_{0}} T_{\vartheta}(F, \vartheta) f_{0, \beta}(\beta) p \\
H(F, \beta, w) & =\rho_{0} \vartheta c_{V}(F, \vartheta) f_{0, \beta}(\beta) w \\
a & =k_{0} \vartheta_{0}, \\
b & =k_{0} \tau_{0},
\end{aligned}
$$

and

$$
\vartheta=\vartheta(\beta, w)=\vartheta_{0} \exp \left\{\frac{1}{\vartheta_{0}}\left(f_{0}(\beta)-\tau_{0} w\right)\right\} .
$$

Note that Eqs. (4.6) imply

$$
\begin{aligned}
\tau_{0} G & =B f_{0, \beta} p, \\
\tau_{0} H & =C f_{0, \beta} w, \\
B & =-\frac{\tau_{0}}{\rho_{0} \vartheta_{0}^{2}} D, \\
c_{V_{, F}} & =-\vartheta T_{\vartheta, \vartheta} .
\end{aligned}
$$

Similarly, as for the rigid conductor, we will consider the one-dimensional acceleration wave $x=\varphi(t)$, across which each of the functions $(v, F, \beta, w, p)$ are continuous, i.e.,

$$
[v]=[F]=[\beta]=[w]=[p]=0,
$$

but the derivatives are not, namely,

$$
\left[v_{, t}\right] \neq 0, \quad\left[F_{, t}\right] \neq 0, \quad\left[p_{, t}\right] \neq 0,
$$

except for the derivative $\beta_{, t},\left[\beta_{, t}\right]=0$ by Eq. $(4.5)_{4}$. 
There are two amplitudes,

$$
\alpha(t)=\left[w_{, t}\right](t) \text { and } \delta(t)=\left[v_{, t}\right](t) .
$$

Evaluating the system (4.5) across the wave (assuming that the coefficients are continuous), and since by Eqs. (3.6) and (4.8)

$$
\begin{array}{rlrl}
{\left[v_{, x}\right]} & =-\frac{1}{s} \delta, & {\left[w_{, x}\right]=-\frac{1}{s} \alpha,} \\
{\left[F_{, t}\right]=-\frac{1}{s} \delta,} & {\left[p_{, t}\right]=-\frac{1}{s} \alpha,} \\
{\left[F_{, x}\right]=\frac{1}{s^{2}} \delta,} & {\left[p_{, x}\right]=\frac{1}{s^{2}} \alpha,}
\end{array}
$$

we find

$$
\begin{aligned}
\left(s^{2}-T_{F}^{+}\right) \delta-B^{+} s \alpha & =0, \\
-D^{+} s \delta+\left(a-C^{+} s^{2}-b p^{+} s\right) \alpha & =0 .
\end{aligned}
$$

The nonzero speeds of the wave satisfy the equation

$$
-C^{+} s^{4}-b p^{+} s^{3}+\left(a+T_{F}^{+} C^{+}-B^{+} D^{+}\right) s^{2}+T_{F}^{+} b p^{+} s-a T_{F}^{+}=0 .
$$

As previously, we assume that the fastest wave is moving into the body that has been in a state of rest, i.e., for $\varphi(t) \leq x$

$$
\begin{aligned}
& \beta(x, t)=\beta^{+}=\text {const }, \quad F(x, t)=F^{+}=\text {const }, \\
& p(x, t)=p^{+}=0, \quad v(x, t)=v^{+}=0, \\
& w(x, t)=w^{+}=0 .
\end{aligned}
$$

In this simplified case, the first wave has a constant speed of propagation $s_{1}$ such that

$$
\left(s_{1}^{2}-T_{F}^{+}\right)\left(a-C^{+} s_{1}^{2}\right)=B^{+} D^{+} s_{1}^{2}<0
$$

and

$$
s_{1}^{2}=\frac{1}{2}\left\{\left(\frac{a}{C^{+}}+T_{F}^{+}-\frac{B^{+} D^{+}}{C^{+}}\right)+\left[\left(\frac{a}{C^{+}}+T_{F}^{+}-\frac{B^{+} D^{+}}{C^{+}}\right)^{2}-4 \frac{a T_{F}^{+}}{C^{+}}\right]^{1 / 2}\right\} \text {. }
$$

We are assuming that $T_{F}^{+}>0$ and $c_{V}^{+}>0$.

For $\tau_{0} \neq 0$, Eq. (4.12) gives the relation between the amplitudes $\delta$ and $\alpha$ :

$$
\delta=\frac{B^{+} s_{1}}{s_{1}^{2}-T_{F}^{+}} \alpha=\frac{a-C^{+} s_{1}^{2}}{D^{+} s_{1}} \alpha .
$$

Next we derive the equation that describes the behaviour of the amplitude $\alpha$ or $\delta$ along the wave with speed $s_{1}^{2}$. As was done in the case of the rigid conductor, we differentiate each equation in system (4.5) with respect to $t$ and then take the jump 
across the wave. To do so, first we recall assumptions (4.8) and (4.9) and relations (4.11); then (cf. Eq. (3.16))

$$
\begin{aligned}
& {\left[v_{, t t}\right]=2 \frac{d}{d t} \delta+s_{1}^{2}\left[F_{, t x}\right],} \\
& {\left[w_{, t t}\right]=2 \frac{d}{d t} \alpha+s_{1}^{2}\left[p_{, t x}\right]}
\end{aligned}
$$

also

$$
\begin{aligned}
& {\left[p_{, t t}\right]=-\frac{1}{s_{1}} \frac{d}{d t} \alpha-s_{1}\left[p_{, t x}\right],} \\
& {\left[F_{, t t}\right]=-\frac{1}{s_{1}} \frac{d}{d t} \delta-s_{1}\left[F_{, t x}\right] .}
\end{aligned}
$$

Since all the coefficients are continuous across the wave, this introduces some simplifications (after Eq. (4.6)):

$$
\begin{array}{ll}
B_{, F}^{+}-T_{F_{, w}}^{+}=2 B_{, F}^{+}, & \tau_{0} H_{, w}^{+}=C^{+} f_{{ }_{, \beta}}^{+}, \\
G_{, w}^{+}=G_{, F}^{+}=H_{, F}^{+}=0, & \tau_{0} G_{, p}^{+}=B^{+} f_{{ }_{, \beta}^{+}}^{+} .
\end{array}
$$

After some lengthy but simple calculations we obtain coupled equations for $\delta$ and $\alpha$ :

$$
\begin{aligned}
\Psi_{1}(\alpha, \delta)+\left(s_{1}^{2}-T_{F}^{+}\right)\left[F_{, x t}\right]-s_{1} B^{+}\left[p_{, t x}\right] & =0, \\
\Psi_{2}(\alpha, \delta)-s_{1} D^{+}\left[F_{, x t}\right]+\left(a-C^{+} s_{1}^{2}\right)\left[p_{, t x}\right] & =0,
\end{aligned}
$$

where we introduced the notation

$$
\begin{aligned}
& \Psi_{1}(\alpha, \delta)=2 \frac{d}{d t} \delta-\frac{B^{+}}{s_{1}} \frac{d}{d t} \alpha+\frac{1}{s_{1}^{3}} T_{F_{, F}}^{+} \delta^{2}-\frac{1}{s_{1}} B_{, w}^{+} \alpha^{2}+\frac{2}{s_{1}^{2}} B_{, F}^{+} \delta \alpha+\frac{1}{s_{1}} \frac{B^{+} f_{0, \beta}^{+}}{\tau_{0}} \alpha, \\
& \Psi_{2}(\alpha, \delta)=-2 C^{+} \frac{d}{d t} \alpha-\frac{D^{+}}{s_{1}} \frac{d}{d t} \delta+\frac{1}{s_{1}^{2}} D_{, F}^{+} \delta^{2}+\left(\frac{1}{s_{1}^{2}} b-C_{, w}^{+}\right) \alpha^{2} \\
& +\frac{1}{s_{1}}\left(C_{, F}^{+}-D_{, w}^{+}\right) \delta \alpha+C^{+} \frac{f_{0, \beta}^{+}}{\tau_{0}} \alpha \text {. }
\end{aligned}
$$

For each of the three cases of function $f_{0}(\beta)$, Eqs. (1.9), (1.10), and (1.11),

$$
\frac{B^{+} f_{0, \beta}^{+}}{\tau_{0}}=-\frac{k_{0}}{k^{+}} T_{\vartheta}^{+},
$$

and

$$
\frac{C^{+} f_{0, \beta}^{+}}{\tau_{0}}=-\frac{\rho_{0} \vartheta_{0} k_{0}}{k^{+}} c_{V}^{+} .
$$

The coefficients in Eq. (4.21) accompanying $\left[F_{, x t}\right]$ and $\left[p_{, x t}\right]$ must satisfy Eq. (4.15); as a consequence we have that $\Psi_{1}$ and $\Psi_{2}$ are proportional,

$$
\Psi_{1}(\alpha, \delta)=\xi \Psi_{2}(\alpha, \delta),
$$


where

$$
\xi=-\frac{s_{1}^{2}-T_{F}^{+}}{s_{1} D^{+}}=-\frac{s_{1} B^{+}}{a-C^{+} s_{1}^{2}} .
$$

This fact together with relation (4.17) leads us to a single equation for $\delta$, again of Bernoulli type,

$$
\frac{d}{d t} \delta+M \delta^{2}+N \delta=0
$$

where the coefficients $\mathscr{M}$ and $\mathscr{N}$ are given by

$$
\begin{aligned}
& \mathscr{M}=\frac{1}{2\left(a T_{F}^{+}-C^{+} s_{1}^{4}\right)}\left[\frac{a-C^{+} s_{1}^{2}}{s_{1}} T_{F, F}^{+}+\frac{s_{1}^{3} B^{+} D^{+}\left(C_{, F}^{+}-2 D_{, w}^{+}\right)}{a-C^{+} s_{1}^{2}}\right. \\
& \left.\quad+3 D^{+} B_{, F}^{+} s_{1}+s_{1} D^{+}\left(b-s_{1}^{2} C_{, w}^{+}\right) \frac{s_{1}^{2}-T_{F}^{+}}{a-C^{+} s_{1}^{2}}\right], \\
& \mathscr{N}=\frac{s_{1}^{2} a^{2}}{\left(a T_{F}^{+}-C^{+} s_{1}^{4}\right)\left(a-C^{+} s_{1}^{2}\right)} \frac{\rho_{0} \vartheta^{+}\left(T_{\vartheta}^{+}\right)^{2}}{2 k^{+}}>0 .
\end{aligned}
$$

The solution of Eq. (4.26) blows up in finite time $t_{1}$ if $\mathscr{M} \delta(0)+\mathscr{N}<0$.

Finally, we would like to investigate some limits concerning the speed of propagation $s$, as a solution of Eq. (4.13). One is for $\tau_{0} \rightarrow 0$, which corresponds to the case of thermoelastic materials with the Fourier law of heat propagation, and the second one, which is hypothetical, for $\tau_{0} \rightarrow \infty$. To do so, let us express explicitly the dependence of the coefficients in Eq. (4.13) on $\tau_{0}$ in the following way:

$$
\mathbf{P}\left(\tau_{0}\right)=-l \tau_{0} s^{4}-m \tau_{0} s^{3}+\left(a+(n l+d) \tau_{0}\right) s^{2}+n m \tau_{0} s-a n=0,
$$

where $l, a, d, m, n$ are positive constants independent on $\tau_{0}$, and from Eqs. (4.6) and (4.7)

$$
\begin{aligned}
l & =\rho_{0} \vartheta^{+} c_{V}^{+}, \\
m & =k_{0} p^{+}, \\
n & =T_{F}^{+}, \\
d & =\rho_{0}\left(\vartheta^{+} T_{\vartheta}^{+}\right)^{2}, \\
a & =k_{0} \vartheta_{0} .
\end{aligned}
$$

For $\tau_{0} \rightarrow 0$, we have $\mathbf{P}(0)=a s^{2}-a n=0$ and $s^{2}=s_{0}^{2}=T_{F}^{+}$, and Eq. (4.26) for the amplitude $\delta$ takes the form derived by Dafermos [5]

$$
\frac{d}{d t} \delta+\frac{T_{F_{F}}^{+}}{2 s_{0}^{3}} \delta^{2}+\frac{\rho_{0} \vartheta^{+}\left(T_{\vartheta}^{+}\right)^{2}}{2 k^{+}} \delta=0 .
$$

If $\tau_{0} \rightarrow \infty$, then from Eq. (4.29),

$$
s\left(-l s^{3}-m s^{2}+(n l+d) s+n m\right)=0
$$

and $s=0$, or else $s$ is a nonzero root of the above polynomial. Since we made assumptions (4.14), the fastest nonzero speed can be obtained with $m=0$, that is,

$$
-l s^{2}+(n l+d)=0
$$


and

$$
s^{2}=s_{\infty}^{2}=n+\frac{d}{l}=T_{F}^{+}+\frac{\vartheta^{+}\left(T_{\vartheta}^{+}\right)^{2}}{c_{V}^{+}}
$$

It is easy to calculate

Proposition 1. If the fastest acceleration wave is moving into a body that has been in a state of rest (4.14), then its speed $s_{1}^{2}$ given by Eq. (4.16), has the following properties:

$$
\begin{gathered}
\lim _{\tau_{0} \rightarrow \infty} s_{1}^{2}=s_{\infty}^{2}, \\
\lim _{\tau_{0} \rightarrow 0} s_{1}^{2}=\infty, \\
s_{1}^{2}>s_{0}^{2}, \\
s_{1}^{2}>s_{\infty}^{2},
\end{gathered}
$$

$s_{1}^{2}$ is a decreasing function of $\tau_{0}$.

As a first approach to discussing the second wave, which generally propagates into a disturbed state with speed $s_{2}$ in the positive direction, we assume that the first

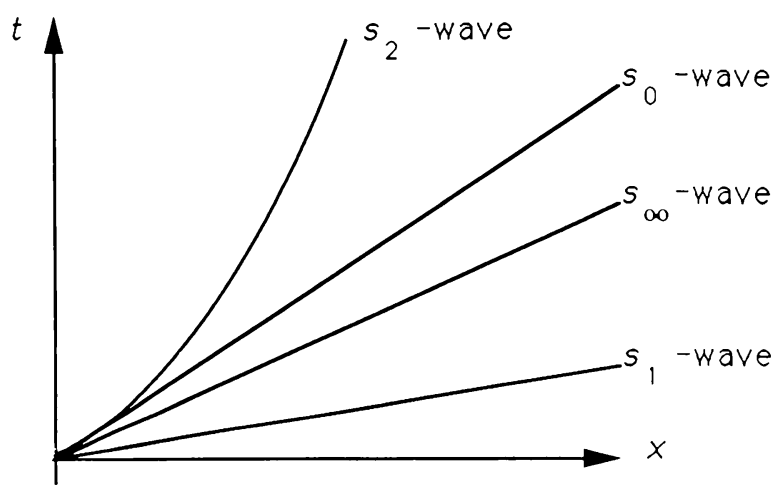

FIGURE 1

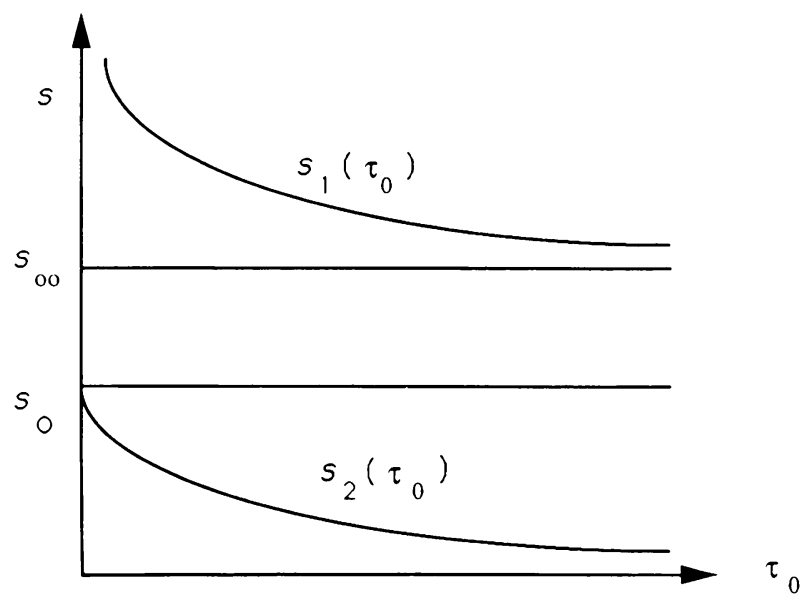

FIGURE 2 
wave did not change the values of $F, v, \beta, w, p$ far from equilibrium. As a consequence, the speed $s_{2}$ can be approximated by (cf. Eq. (4.16)) the formula

$$
s_{2}^{2}=\frac{1}{2}\left\{\left(\frac{a}{C^{+}}+T_{F}^{+}-\frac{B^{+} D^{+}}{C^{+}}\right)-\left[\left(\frac{a}{C^{+}}+T_{F}^{+}-\frac{B^{+} D^{+}}{C^{+}}\right)^{2}-4 \frac{a T_{F}^{+}}{C^{+}}\right]^{1 / 2}\right\} \text {. }
$$

Proposition 2. If the second wave propagates into a state close to equilibrium and is again given by Eq. (4.39), then

$$
\begin{gathered}
\lim _{\tau_{0} \rightarrow \infty} s_{2}^{2}=0, \\
\lim _{\tau_{0} \rightarrow 0} s_{2}^{2}=s_{0}^{2}=T_{F}^{+}, \\
s_{2}^{2}<s_{0}^{2}, \\
s_{2}^{2}<s_{\infty}^{2},
\end{gathered}
$$

$s_{2}^{2}$ is a decreasing function of $\tau_{0}$.

The analysis performed so far allows us to sketch (Fig. 1) in the phase plane the following representation of the wave fronts for positive $s_{1}$ and $s_{2}$.

Here, $s_{2}$ is the speed of the slower wave propagated into the disturbed region. However, in terms of $\tau_{0}$, regarded as a variable we can sketch the graph of $s_{1}$ and $s_{2}$ as functions of $\tau_{0}$ (Fig. 2).

It follows from both figures and the propositions that, in the limit of vanishing $\tau_{0}$, the larger characteristic speed $s_{1}$ goes to infinity, while the smaller one $s_{2}$ tends to the classical thermoelastic wave speed $s_{0}$ obtained by Dafermos [5] and by others.

Acknowledgment. The paper was completed when the first author was an appointed Visiting Professor at Loyola University.

\section{REFERENCES}

[1] M. Chester, Second sound in solids, Phys. Rev. 131, 2013-2015 (1962)

[2] V. A. Cimmelli and W. Kosiński, Well posedness results for a nonlinear hyperbolic heat equation, submitted

[3] B. C. Coleman and M. E. Gurtin, Waves in materials with memory, II. On the growth and decay of one-dimensional acceleration waves, Arch. Rational Mech. Anal. 19, 239-265 (1965)

[4] B. C. Coleman and M. E. Gurtin, Waves in materials with memory, III. Thermodynamic influence on the growth and decay of acceleration waves, Arch. Rational Mech. Anal. 19, 266-298 (1965)

[5] C. M. Dafermos, Contemporary issues in the dynamic behaviour of continuous media, LCDS Lecture Notes, Brown University, vol. 85-1, 1985

[6] C. M. Dafermos and L. Hsiao, Development of singularities in solutions of the equations of nonlinear thermoelasticity, Quart. Appl. Math. 44, 463-474 (1986)

[7] M. E. Gurtin and A. C. Pipkin, A general theory of heat conduction with finite wave speeds, Arch. Rational Mech. Anal. 31, 113-126 (1968)

[8] W. Kosinski, Elastic waves in the presence of a new temperature scale, Elastic wave propagation, M. F. McCarthy and M. A. Hayes, eds., Elsevier Science (North-Holland), Amsterdam, 1989, p. 629

[9] P. D. Lax, Development of singularities of solutions of nonlinear hyperbolic partial differential equations, J. Math. Phys. 5, 611-613 (1964)

[10] M. Slemrod, Global existence, uniqueness and asymptotic stability of classical smooth solutions in one-dimensional, non-linear thermoelasticity, Arch.Rational Mech. Anal. 76, 97-133 (1981) 